\section{REVISTA BRASILEIRA DE QUALIDADE DE VIDA}

\title{
Diferença nas condições de saúde de deficientes físicos ativos e sedentários
}

\section{Difference in health conditions of active and sedentary disabled persons}

\author{
Juliana Lima Valério \\ Universidade Estadual do Centro-Oeste - UNICENTRO - Guarapuava - Paraná - Brasil \\ julimavalerio@gmail.com \\ Aline Cristina Carrasco \\ Universidade Estadual do Centro-Oeste - UNICENTRO - Guarapuava - Paraná - Brasil \\ alineccarrasco@gmail.com \\ Andersom Ricardo Fréz \\ Universidade Estadual do Centro-Oeste - UNICENTRO - Guarapuava - Paraná - Brasil \\ andersom_frez@yahoo.com.br
}

\section{RESUMO}

OBJETIVO: Avaliar a influência da prática de atividade física nas variáveis qualidade de vida, independência funcional e presença de sintomatologia dolorosa em indivíduos com deficiência física da Associação de Deficientes Físicos de Guarapuava (ADFG).

MÉTODOS: 35 indivíduos com deficiência física foram divididos em dois grupos: ativo $(\mathrm{n}=14)$ e sedentário $(\mathrm{n}=21)$. A qualidade de vida foi avaliada pela versão abreviado do World Health Organization Quality of Life Instruments (WHOQOL-Bref), a independência funcional pelo Índice de Barthel e a prevalência de sintomas musculoesqueléticos pelo Questionário Nórdico de Sintomas Osteomusculares. Todos os indivíduos foram avaliados durante as reuniões da ADFG. Para análise dos resultados utilizou-se o teste t não pareado para as amostras com distribuição normal, e o teste não paramétrico de Mann-Whitney para as amostras com hipótese de normalidade rejeitada. O nível de significância foi estabelecido em $\mathrm{p}<0,05$.

RESULTADOS: Não foram observadas diferenças estatísticas entre os grupos em todas as variáveis avaliadas.

CONCLUSÕES: Para a amostra de deficientes físicos avaliada não foram observadas diferenças significativas na qualidade de vida, independência funcional e presença de sintomatologia dolorosa entre os indivíduos ativos e os sedentários.

PALAVRAS-CHAVE: Pessoas com deficiência. Exercício. Estilo de vida sedentário. Nível de saúde. Qualidade de vida. 


\section{ABSTRACT}

OBJECTIVE: To evaluate the influence of physical activity on quality of life, functional independence and prevalence of musculoskeletal symptoms of disabled person of Physical Disabled Association from Guarapuava, Brazil.

METHODS: 35 individual with physical disabilities were divided into two groups: active $(\mathrm{n}=14)$ and sedentary $(n=21)$. Quality of life was assessed by the brief version of World Health Organization Quality of Life Instruments (WHOQOL-Brief), the functional independence by the Barthel Index and the prevalence of musculoskeletal symptoms by the Nordic Musculoskeletal Questionnaire. All subjects were evaluated during the association meeting. To analyze the results we used the unpaired t test for samples with normal distribution, and the nonparametric MannWhitney for samples having reject normality hypothesis. The significance level was $\mathrm{p}<0.05$.

RESULTS: No statically differences were observed between groups in all variables.

CONCLUSIONS: For this sample of disabled persons no significant differences were observed in quality of life, functional independence and painful symptoms among active and sedentary individuals.

KEYWORDS: Disabled persons. Exercise. Sedentary lifestyle. Health status. Quality of life.

\section{Introdução}

Os resultados do Censo Demográfico de 2010 mostraram que, no Brasil, mais de 45 milhões de pessoas apresentam algum tipo de deficiência, correspondendo a quase $24 \%$ da população brasileira e dessas, mais de 13 milhões apresentam deficiência motora (INSTITUTO BRASILEIRO DE GEOGRAFIA E ESTATÍSTICA, 2010). Porém, o processo incapacitante de cada sujeito é representado por dimensões distintas (CASTRO et al., 2008; SAMPAIO; LUZ, 2009).

No contexto da reabilitação, um dos principais objetivos é atingir a independência funcional (BARFIELD; MALONE, 2013; SILVA, 2011). Porém, mesmo na presença de incapacidades, as pessoas podem equilibrar suas limitações com suas potencialidades e viver com qualidade de vida, mesmo tendo uma deficiência (RESENDE; GOUVEIA, 2011).

Um dos fatores que pode contribuir para a qualidade de vida é a prática de atividade física, por meio de adaptações fisiológicas e por ser um mecanismo facilitador na inclusão pessoas na sociedade (ASA; GARCIA; MATUTI, 2015; CARROLL et al., 2014; NOCE; SIMIM; MELLO, 2009). O esporte adaptado tem proporcionado oportunidades para as pessoas com deficiência, e esta prática vem crescendo nos serviços de reabilitação (BARFIELD; MALONE, 2013; SILVA, 2011).

Dessa forma, o objetivo desta pesquisa foi avaliar a influência da prática de atividade física nas variáveis qualidade de vida, independência funcional e presença de sintomatologia dolorosa em indivíduos com deficiência física da Associação de Deficientes Físicos de Guarapuava (ADFG).

\section{Métodos}

Esta pesquisa caracterizou-se como um estudo transversal com indivíduos da ADFG. O estudo foi realizado entre agosto e outubro de 2014, e foi aprovado pelo Comitê de Ética em Pesquisa da Universidade Estadual do Centro-Oeste, parecer 344.443/2013, obedecendo a Resolução 466/2012 do Conselho Nacional de Saúde.

Fazem parte da ADFG 150 indivíduos cadastrados e destes, 50 participam ativamente da associação, os quais foram convidados para integrar a amostra da pesquisa. Foram incluídos os indivíduos com disfunções do aparelho locomotor, independente da etiologia, e excluídos os que apresentavam dificuldade para compreensão verbal e/ou escrita. 35 indivíduos enquadraram-se nos 
critérios estabelecidos, e todos receberam os esclarecimentos do estudo e assinaram um termo de consentimento.

Os indivíduos foram divididos em dois grupos: ativo (GA), composto por 14 indivíduos que praticavam basquete em cadeira de rodas, e grupo sedentário (GS), constituído por 21 indivíduos sedentários.

Foram consideradas como variáveis a qualidade de vida, a independência funcional e a prevalência de sintomas musculoesqueléticos.

Para avaliar a qualidade de vida foi utilizada a versão abreviada do World Health Organization Quality of Life Instruments (WHOQOL-Bref), composta por 26 questões que compõem 4 domínios: físico, psicológico, relações sociais e meio ambiente (FLECK et al., 2000).

Para avaliar a independência funcional foi utilizado o Índice de Barthel. Esta escala avalia as atividades de vida diária e mede a independência funcional no cuidado pessoal, mobilidade, locomoção e eliminações. Cada item é pontuado de acordo com o desempenho do indivíduo em realizar tarefas de forma independente, com alguma ajuda ou de forma dependente. O indivíduo é classificado nos níveis funcionais: dependência total, severa, moderada, leve e totalmente independente (MINOSSO et al., 2010; SOUZA et al., 2013).

Para avaliar a prevalência de sintomas musculoesqueléticos foi utilizado o Questionário Nórdico de Sintomas Osteomusculares (QNSO), composto por uma figura humana dividida em nove regiões anatômicas: região cervical, ombros, região torácica, cotovelos, punhos/mãos, região lombar, quadril/coxas, joelhos e tornozelos/pé. Apresenta questões relacionadas com cada área anatômica citada e verifica se os respondentes tiveram dores nos últimos 12 meses e nos últimos 7 dias (PINHEIRO; TRÓCCOLI; CARVALHO, 2002).

A coleta de dados aconteceu durante as reuniões da ADFG.

Foi realizada análise estatística descritiva para calcular a média e o desvio padrão dos resultados. Para a comparação dos resultados dos questionários empregou-se o teste de normalidade Kolmogorov-Smirnov e a distribuição foi considerada normal se $p>0,05$. Para a detecção entre as variáveis utilizou-se o teste $\mathrm{t}$ não pareado para as amostras com distribuição normal, e o teste não paramétrico de Mann-Whitney para as amostras com hipótese de normalidade rejeitada. O nível de significância foi estabelecido em $\mathrm{p}<0,05$. Os testes foram realizados com o uso do software Graphpad Instat versão 3.0.

\section{Resultados}

A Tabela 1 apresenta as características dos indivíduos de cada grupo: 
Tabela 1 - Características dos grupos

\begin{tabular}{|c|c|c|}
\hline Variárvel & GA $(n=14)$ & GS $(n=21)$ \\
\hline Idade (anos) média \pm DP & $35,6 \pm 15,7$ & $43,8 \pm 13,6$ \\
\hline Sexo - Masculino/Feminino, $\mathrm{n}(\%)$ & $14(100,0) /-$ & $14(66,7) / 7(33,3)$ \\
\hline \multicolumn{3}{|l|}{ Estado civil, $\mathrm{n}(\%)$} \\
\hline Solteiro & $4(28,6)$ & $12(57,2)$ \\
\hline Casado & $10(71,4)$ & $4(19,0)$ \\
\hline Viúvo & - & $2(9,5)$ \\
\hline Outro & - & $3(14,3)$ \\
\hline \multicolumn{3}{|l|}{ Escolaridade, $\mathrm{n}(\%)$} \\
\hline Sem escolaridade & $1(7,1)$ & $3(14,3)$ \\
\hline Ensino fundamental incompleto & $4(28,6)$ & $7(33,3)$ \\
\hline Ensino fundamental completo & $4(28,6)$ & $3(14,3)$ \\
\hline Ensino médio completo & $1(7,1)$ & $7(33,3)$ \\
\hline Ensino superior completo & $4(28,6)$ & $1(4,8)$ \\
\hline \multicolumn{3}{|l|}{ Fonte de renda } \\
\hline Trabalham & $2(14,3)$ & $2(9,5)$ \\
\hline Beneficiário & $8(57,1)$ & $17(81,0)$ \\
\hline Dependente de terceiros & $4(28,6)$ & $2(9,5)$ \\
\hline \multicolumn{3}{|l|}{ Tipo de deficiência, $\mathrm{n}(\%)$} \\
\hline Amputação & $3(21,5)$ & $2(9,5)$ \\
\hline Traumatismo raquimedular & $6(43,0)$ & $3(14,3)$ \\
\hline Paralisia cerebral & $1(7,1)$ & $3(14,3)$ \\
\hline Mielomeningocele & $1(7,1)$ & - \\
\hline Acidente vascular encefálico & $1(7,1)$ & $1(4,8)$ \\
\hline Poliomielite & $1(7,1)$ & $10(47,5)$ \\
\hline Má formação congênita & - & $1(4,8)$ \\
\hline Artrite reumatóide & - & $1(4,8)$ \\
\hline Outros & $1(7,1)$ & - \\
\hline \multicolumn{3}{|l|}{ Etiologia, $\mathrm{n}(\%)$} \\
\hline Adquirida & $12(85,7)$ & $17(81,0)$ \\
\hline Congênita & $2(14,3)$ & $4(19,0)$ \\
\hline Tempo de deficiência (anos) média \pm DP & $23,0 \pm 17,0$ & $9,6 \pm 15,1^{*}$ \\
\hline
\end{tabular}
$(\mathrm{p}=0,47)$.

A funcionalidade avaliada pelo Índice de Barthel (Tabela 2) foi semelhante entre os grupos

Tabela 2 - Categorias do Índice de Barthel

\begin{tabular}{lcccc}
\hline & \multicolumn{2}{c}{$\mathbf{G A}(\mathbf{n}=\mathbf{1 4})$} & \multicolumn{2}{c}{$\mathbf{G S}(\mathbf{n}=\mathbf{2 1})$} \\
& Indice & $\mathbf{n}$ & Índice & $\mathbf{n}$ \\
\hline Dependência leve & 78,1 & 9 & 80,7 & 15 \\
Dependência moderada & 65,3 & 4 & 70,4 & 5 \\
Dependência severa & 49,0 & 1 & - & - \\
Dependência total & - & - & 21 & 1 \\
\hline Fonte: Autoria própria (2015). & \multicolumn{4}{l}{}
\end{tabular}

Com relação à qualidade de vida, a média dos domínios do WHOQOL-Bref dos grupos GA e GS não demonstrou diferença significativa (Tabela 3). Os escores dos domínios estão apresentados no Gráfico 1. 
Tabela 3 - Diferença na qualidade de vida entre os deficientes físicos ativos e os sedentários

\begin{tabular}{lccc}
$\begin{array}{c}\text { Domínios } \\
\text { WHOQOL-bref }\end{array}$ & $\begin{array}{c}\text { Ativo } \\
(\mathbf{n = 1 4 )}\end{array}$ & $\begin{array}{c}\text { Sedentário } \\
(\mathbf{n = 2 1})\end{array}$ & p-valor \\
\hline Físico & $3,4 \pm 1,0$ & $3,1 \pm 1,3$ & 0,1512 \\
Psicológico & $3,7 \pm 1,0$ & $3,7 \pm 1,1$ & 0,6368 \\
Relações sociais & $3,7 \pm 0,9$ & $3,7 \pm 1,1$ & 0,8148 \\
Meio ambiente & $3,6 \pm 1,0$ & $3,7 \pm 1,0$ & 0,5368 \\
\hline
\end{tabular}

Fonte: Autoria própria (2015).

Gráfico 1 - Escores (em \%) dos domínios do WHOQOL-Bref, grupo ativo (GA) e grupo sedentário (GS)

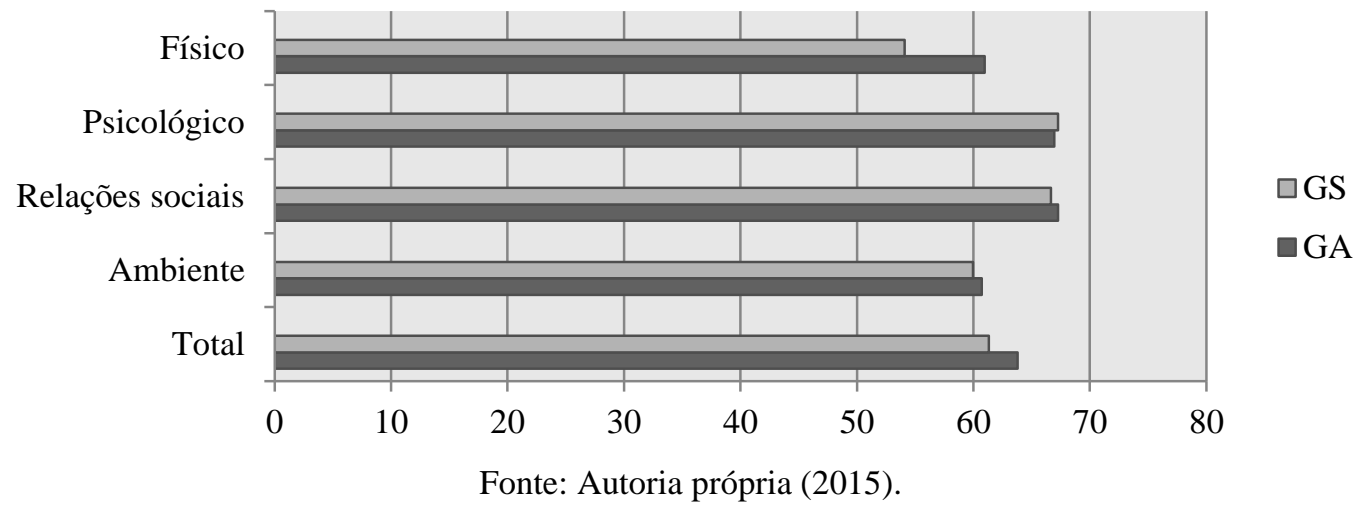

Com relação aos sintomas musculoesqueléticos, no GA 78,6\% (n=11) dos indivíduos referiram sintomas nos últimos 12 meses, sendo as principais queixas na coluna torácica, lombar, ombro e punho. Destes, $28,6 \%(\mathrm{n}=4)$ possuíam algum sintoma no momento da entrevista e 50,0\% $(n=7)$ referiram algum sintoma que lhes impediu de realizar atividades de vida diária nos últimos 12 meses, com necessidade de procurar o serviço de saúde devido ao sintoma. No GS 85,7\% ( $\mathrm{n}=18$ ) apresentaram algum sintoma no período de 12 meses, com queixas de sintomatologia dolorosa na coluna cervical, torácica e lombar e punho. No momento da entrevista, 76,2\% $(n=16)$ apresentaram algum sintoma nos últimos 12 meses, 42,9\% $(\mathrm{n}=9)$ foram impedidos de realizar suas atividades diárias devido a algum sintoma musculoesquelético e 61,9\% (n=13) necessitaram procurar serviço de saúde.

\section{Discussão}

O trauma é umas das causas mais comuns de lesão da medula espinal (MARTINS; RABELO, 2008) e na faixa etária de 21 a 30 anos, há um predomínio de traumas decorrentes de acidentes automobilísticos e quedas de altura (CAMPOS et al., 2008; TEE et al., 2013). No presente estudo, as principais causas de deficiência física no GA foram as lesões decorrentes de acidentes automobilísticos e quedas, envolvendo adultos jovens com idade média de 23 anos. Já no GS, a maior incidência de deficiência física estava relacionada às doenças congênitas e a poliomielite. Observou-se que os indivíduos com lesões adquiridas foram os que mais procuraram os esportes. Acredita-se que por serem os indivíduos que adquiriram a deficiência na juventude, ou ainda pela tentativa de melhorar os aspectos psicológicos para melhor aceitação da deficiência. Tal fato sugere que quem adquiriu a deficiência na infância ou mesmo de forma congênita está mais acomodado ou já aceitou a condição em que vive e, por isso, não se sente motivado ou interessado em procurar atividade física. Assim, é importante a inclusão de políticas públicas que incentivem as pessoas com deficiência física a praticarem alguma atividade física, como também para melhorar as condições do ambiente físico permitindo a prática de esportes adaptados. 
A realização das atividades de vida diária pode ser afetada por diversos fatores, como a diminuição da capacidade funcional, que interfere na independência do indivíduo (SILVA, 2011). No presente estudo ambos os grupos apresentaram comprometimentos funcionais com grau de dependência leve. Os maiores comprometimentos e limitações observados estavam relacionados às tarefas de subir e descer escadas no GA e cadeira de rodas e transferências no GS. Entretanto, não foram observadas diferenças significativas entre os grupos. Esperava-se que o GA apresentasse limitações menores em relação ao GS, pois as pessoas com deficiência que realizam atividades físicas apresentam melhor capacidade física, função cardiorrespiratória e maior força muscular, que proporcionam melhores habilidades funcionais, fazendo com que obtenham melhores resultados no âmbito da funcionalidade (FURMANIUK; WASILEWSKA; KACZMAREK, 2010; MARTINS; RABELO, 2008). Um dos possíveis fatores que pode ter influência nesse resultado foi que em ambos os grupos mais de $64,0 \%$ dos indivíduos foram classificados como 'dependência leve' pelo Índice de Barthel.

$\mathrm{Na}$ análise da qualidade de vida também não foram observadas diferenças significativas entre os grupos. Resultados diferentes de outros estudos que também utilizaram o WHOQOL-Bref para comparar indivíduos com deficiência física ativos e sedentários (ANTONIETTI et al. 2008; LIMA; RIBEIRO; TONELLO, 2012; NOCE; SIMIM; MELLO, 2009). No trabalho de Noce, Simim e Mello (2009), com 20 indivíduos com deficiência física, o GA obteve maiores escores em todos os domínios em relação ao GS, indicando melhor qualidade de vida para o GA. Já no estudo realizado por Antonietti et al. (2008), com 27 indivíduos com lesão da medula espinal, o GA obteve melhores escores nos aspectos físicos $(p=0,001)$, aspectos psicológicos $(p=0,002)$ e relações pessoais ( $\mathrm{p}=0,026)$. No estudo de Lima, Ribeiro e Tonello (2012), com 100 pessoas com deficiência física, os resultados apontaram que os indivíduos que realizavam atividades de lazer apresentaram diferenças estatísticas significantes em todos os domínios de qualidade de vida: físico, psicológico, relações sociais e meio ambiente. Os maiores índices encontrados foram nos domínios psicológico $(81,2 \%)$ e social $(82,6 \%)$ e os menores no domínio físico $(71,4 \%)$ e ambiente $(67,8 \%)$.

A influência dos fatores ambientais também é citada por Carroll et al. (2014). Os autores relatam que existem barreiras que limitam as pessoas com deficiência para a prática de atividades físicas. Essas barreiras incluem limitações no ambiente construído ou natural (falta de acessibilidade), limitações físicas, limitações emocionais e falta de programas de atividade física com instalações acessíveis a essa população.

$\mathrm{Na}$ questão das relações sociais e aspectos psicológicos, Ferreira et al. (2011) apontam a existência de efeitos positivos da prática regular de atividade física para a saúde mental, em diferentes indicadores, tais como os estados de humor, a depressão, a ansiedade, o estresse e a autoestima. Tratando-se de pessoas com deficiência, a prática de atividades físicas é de fundamental importância, pois gera benefícios na interação social quando se pratica um esporte em equipe, benefícios físicos incluindo a melhoria do tônus muscular e funcionamento cardiovascular e benefícios psicológicos afetando positivamente a qualidade de vida dessas pessoas (BARFIELD; MALONE, 2013). Entretanto, alguns benefícios não foram observados no presente estudo, enquanto os outros não foram avaliados.

Com relação aos sintomas musculoesqueléticos, o presente estudo mostrou que a coluna cervical, torácica e lombar, de maneira geral, parece ser o local em que mais ocorrem lesões musculoesqueléticas nos deficientes físicos, e no GA há também a prevalência de sintomas em ombro e punho. Os resultados são semelhantes aos relatados por Costa et al. (2012). Costa et al. (2012) constataram que os sintomas dolorosos em atletas do basquetebol em cadeira de rodas predominavam na coluna vertebral, mas estavam associados aos sintomas em membros superiores e inferiores. Ainda com atletas do basquetebol em cadeira de rodas, Pereira e Gabriel (2006) constataram que as regiões mais acometidas foram ombro e coluna lombar. Já Antonietti et al. (2008) verificaram que o ombro foi a região de maior prevalência de lesões musculoesqueléticas tanto nos indivíduos sedentários quanto nos ativos. No presente estudo acredita-se que a prevalência de sintomatologia dolorosa na coluna vertebral, ombro e punho esteja associada ao uso da cadeira de rodas na prática esportiva, pois descarregam peso na posição sentada e utilizam os membros 
superiores para arremessos, recepções e rebotes de bola e, ao mesmo tempo, para a propulsão da cadeira de rodas com objetivo de deslocamento em quadra e locomoção. A posição sentada aumenta em 40,0\% a pressão no disco intervertebral em relação à posição em pé, transferindo o peso para os ísquios não havendo o amortecedor dos membros inferiores para fazer a descarga de peso. Quanto maior a pressão sobre os ísquios, maior a compressão na coluna vertebral, além dos movimentos de abaixar, inclinar, rodar e também ao cair que se tornam lesivos para a coluna vertebral (PEREIRA; GABRIEL, 2006). No GS, possivelmente, a prevalência de sintomatologia dolorosa esteja associada ao uso de cadeira de rodas ou outros dispositivos auxiliares, como muletas e andadores, no dia a dia, para locomoção e transferência. O uso desses dispositivos também gera danos e desconfortos nos membros superiores e coluna vertebral, pois algumas atividades de vida diária, como a propulsão da cadeira de rodas e as transferências requerem altas forças e repetitividade de movimentos nos membros superiores (MANDY; REDHEAD; MICHAELIS, 2014; OJEDA; DING, 2014). Tsai et al. (2014) observaram que as transferências são as maiores causas de lesões nos membros superiores, devido ao posicionamento das articulações e a alta força gerada para impulsão.

Este estudo limitou-se a uma amostra de pessoas com deficiência física que frequentavam uma associação da cidade de Guarapuava-PR, o que não permite a generalização dos resultados.

\section{Conclusão}

Para a amostra de deficientes físicos avaliada não foram observadas diferenças significativas na qualidade de vida, independência funcional e presença de sintomatologia dolorosa entre os indivíduos ativos e os sedentários.

\section{Referências}

ANTONIETTI, L. S.; COSTA, R. A.; GONDO, F. L. B.; OLIVEIRA, A. S. B.; CHIARELLO, B. Avaliação comparativa em lesados medulares sedentários e praticantes de basquetebol em cadeira de rodas. Revista de Neurociências, São Paulo, v. 16, n. 2, p. 90-96, 2008.

ASA, S. K. P.; GARCIA, J. R. O.; MATUTI, G. S. Efeitos de um programa de condicionamento físico na qualidade de vida e funcionalidade em indivíduos com sequelas de um acidente vascular encefálico. Revista Brasileira de Qualidade de Vida, Ponta Grossa, v. 7, n. 2, p. 65-74, abr./jun. 2015.

BARFIELD, J. P.; MALONE, L. A. Perceived exercise benefits and barriers among power wheelchair soccer players. Journal of Rehabilitaton Research and Development, Whashington, v. 50, n. 2, p. 231-238, 2013.

CAMPOS, M. F.; RIBEIRO, A. T.; LISTIK, S.; PEREIRA, C. A. B.; SOBRINHO J. A.; RAPOPORT, A. Epidemiologia do traumatismo da coluna vertebral. Revista do Colégio Brasileiro de Cirurgiões, Rio de Janeiro, v. 35, n. 2, p. 88-93, mar./abr. 2008.

CARROLL, D. D.; LONG, E. A. C.; STEVENS, A. C.; SLOAN, M. L.; LULLO, C.; VISSER, S. N.; FOX, M. H.; ARMOUR, B. S.; CAMPBELL, V. A.; BROWN, D. R.; DORN, J. M. Vital signs: disability and physical activity - United States, 2009-2012. Morbidity and Mortality Weekly Report, Atlanta, v. 63, n. 18, p. 407-413, May 2014. 
CASTRO, S. S.; CÉSAR, C. L. G.; CARANDINA, L.; BARROS, M. B. A.; ALVES, M. C. G. P. GOLDBAUM, M. Deficiência visual, auditiva e física: prevalência e fatores associados em estudo de base populacional. Cadernos de Saúde Pública, Rio de Janeiro, v. 24, n. 8, p. 1773-1782, ago. 2008.

COSTA, S. M. L.; BARROS, A. A.; SITONIO, F. T.; BARROS, M. F. A.; CARVALHO, A. G. C. Perfil do atleta de basquetebol sobre rodas de uma instituição pública. Revista Brasileira de Ciências da Saúde, João Pessoa, v. 16, n. 2, p. 59-64, 2012. Grossef

FERREIRA, J. P.; GASPAR, P. M.; CAMPOS, M. J.; SERRA, C. M. Auto-eficácia, competência física e auto-estima em praticantes de basquetebol com e sem deficiência física. Motricidade, Vila Real, v. 7, n. 1, p. 55-68, 2011.

FLECK, M. P. A.; LOUZADA, S.; XAVIER, M.; CHACHAMOVICH, E.; VIEIRA, G.; SANTOS, L.; PINZON, V. Aplicação da versão em português do instrumento abreviado de avaliação da qualidade de vida 'WHOQOL-bref'. Revista Saúde Pública, São Paulo, v. 34, n. 2, p. 178-183, abr. 2000. crossef

FURMANIUK, L.; WASILEWSKA, G. C.; KACZMAREK, D. Influence of long-term wheelchair rugby training on the functional abilities of persons with tetraplegia over a two-year period postspinal cord injury. Journal of Rehabilitation Medicine, Stockholm, v. 42, n. 7, p. 688-690, July 2010. Crossef

INSTITUTO BRASILEIRO DE GEOGRAFIA E ESTATÍSTICA (IBGE). Censo Demográfico 2010: características gerais da população, religião e pessoas com deficiência. Rio de Janeiro: IBGE, 2010. Disponível em:

〈http://biblioteca.ibge.gov.br/visualizacao/periodicos/94/cd_2010_religiao_deficiencia.pdf $>$. Acesso em: 10 jan. 2015.

LIMA, R. M. C.; RIBEIRO, P. R. Q.; TONELLO, M. G. M. Percepção da qualidade de vida por pessoas com deficiências físicas praticantes e não praticantes de atividades de lazer. Licere (Online), Belo Horizonte, v. 15, n. 4, p. 1-14, dez. 2012.

MANDY, A.; REDHEAD, L.; MICHAELIS, J. Measurement of hand/handrim grip forces in two different one arm drive wheelchairs. BioMed Research International, New York, v. 2014, Article ID 509898, 2014.

MARTINS, D. L.; RABELO, R. J. Influência da atividade física adaptada na qualidade de vida de deficientes físicos. Movimentum Revista Digital de Educação Física, Iopatinga, v. 2, n. 2, p. 111, ago./dez. 2008.

MINOSSO, J. S. M.; AMENDOLA, F.; ALVARENGA, M. R. M.; OLIVEIRA, M. A. C. Validação, no Brasil, do Índice de Barthel em idosos atendidos em ambulatórios. Acta Paulista de Enfermagem, São Paulo, v. 23, n. 2, p. 218-223, mar./abr. 2010. crossef

NOCE, F; SIMIM, M. A. M.; MELLO, M. T. A percepção da qualidade de vida de pessoas portadoras de deficiência física pode ser influenciada pela pratica de atividade física? Revista Brasileira de Medicina do Esporte, Niterói, v. 15, n. 3, p. 174-178, maio/jun. 2009.

OJEDA, M.; DING, D. Temporal parameters estimation for wheelchair propulsion using wearable sensors. BioMed Research International, New York, v. 2014, Article ID 645289, 2014. 
PEREIRA, L. C.; GABRIEL, M. G. Incidência de lesões desportivas nos atletas do basquete em cadeira de rodas. 2006. 46 f. Trabalho de conclusão de curso. (Graduação em Fisioterpaia) Universidade da Amazônia, Belém, 2006.

PINHEIRO, F. A.; TRÓCCOLI, B. T.; CARVALHO, C. V. Validação do Questionário Nórdico de Sintomas Osteomusculares como medida de morbidade. Revista Saúde Pública, São Paulo, v. 36, n. 3, p. 307-312, jun. 2002. Crossef

RESENDE, M. C.; GOUVEIA, V. V. Qualidade de vida em adultos com deficiência física. Psicologia: Reflexão e Crítica, Porto Alegre, v. 24, n. 1, p. 99-106, 2011. Crossef

SAMPAIO, R. F.; LUZ, M. T. Funcionalidade e incapacidade humana: explorando o escopo da classificação internacional da Organização Mundial de Saúde. Cadersos de Saúde Pública, Rio de Janeiro, v. 25, n. 3, p. 475-483, mar. 2009.

SILVA, R. A. Condições de funcionalidade de pessoas com lesão medular fundamentadas no índice de Barthel: proposta de intervenção de enfermagem. 2011. 103 f. Dissertação (Mestrado em Enfermagem) - Universidade Federal do Ceará, Fortaleza, 2011.

SOUZA, L. P.; BILATI, I. S.; PRESTES, M. F.; LIMA, A. A. M. Avaliação do Índice de Barthel aplicado ao deficiente físico. Revista Uningá, Maringá, v. 37, p. 13-34, jul./set. 2013.

TEE, J. W.; CHAN, C. H. P.; FITZGERALD, M. C. B.; LIEW, S. M.; ROSENFELD, J. V. Epidemiological trends of spine trauma: an Australian level 1 trauma centre study. Global Spine Journal, New York, v. 3, n. 2, p. 75-84, June 2013. rossef

TSAI, C. Y.; HOGABOOM, N. S.; BONINGER, M. L.; KOONTZ, A. M. The relationship between independent transfer skills and upper limb kinetics in wheelchair users. BioMed Research International, New York, v. 2014, Article ID 984526, 2014. 